\title{
THE EXISTENCE OF AN EXTREMUM IN PROBLEMS OF MAYER*
}

BY

\author{
LAWRENCE M. GRAVES
}

The problem of Mayer with variable end points as stated by Bliss $\dagger$ is that of finding in a class of arcs

$$
y_{i}=y_{i}(x) \quad(a \leqq x \leqq b ; i=1, \cdots, n)
$$

satisfying a system of differential equations and end conditions

$$
\phi_{\alpha}\left(x, y, y^{\prime}\right)=0, \quad \psi_{\mu}[a, b, y(a), y(b)]=0,
$$

one which minimizes a function $g[a, b, y(a), y(b)]$. All simple integral problems of the calculus of variations for which a fairly complete theory of the relative extremum problem has been developed may be transformed to a problem of the above type. The theorems we shall prove concerning the existence of an absolute minimum may be translated directly into corresponding existence theorems for the problem of Bolza or for the problem of Lagrange. The problem of Mayer is considered here because it seems notationally simpler than the problem of Bolza.

An existence theorem for a complicated problem such as we are considering must naturally impose rather severe restrictions on the differential equations involved. In order to treat as wide a variety of cases as possible, the variable functions are divided into groups satisfying different types of conditions. We shall divide them notationally into two groups. The functions $y_{i}(x)$, defined for $a \leqq x \leqq b$, will be regarded as independent functions, and the curve they determine in $x y$-space will be denoted by $C$. When the functions $y_{i}(x)$ are absolutely continuous, dependent functions $z_{\sigma}(x)$ will be determined by "differential equations" and initial conditions of the special form

$$
z_{\sigma}^{\prime}=h_{\sigma}\left(x, y, y^{\prime}, z\right), \quad z_{\sigma}(a)=\zeta_{\sigma} .
$$

A solution of these equations, when existent, is a function of the curve $C$ and the initial values $\zeta$, and will be denoted by $z_{\sigma}=z_{\sigma}[x ; C, \zeta]$. Other types of equations and inequalities affecting the functions $y_{i}(x)$ only are introduced in $\$ 4$. The principal change these additional conditions make is to permit a weakening of the hypotheses.

* Presented to the Society, April 15, 1933; received by the editors September 3, 1935.

$\dagger$ The problem of Mayer with variable end points, these Transactions, vol. 19 (1918), pp. 305-314.

$\ddagger$ Compare Graves, On the existence of the absolute minimum in problems of Lagrange, Bulletin of the American Mathematical Society, vol. 39 (1933), pp. 101-104. 
The final theorems state the existence of a minimum for a function $I(C, \zeta)=g[a, b, y(a), y(b), \zeta, z(b)]$ in a quasi-closed class of admissible elements $(C, \zeta)$. The end conditions do not enter the theorems explicitly. But the nature of a quasi-closed class implies that in general the end conditions $\psi_{\mu}=0$ do not involve the end values $z(b)$.

The proof in $\S 1$ of the lower semi-continuity of the functionals $z[x ; C, \zeta]$ defined by the equations (1) was suggested to me by McShane. This suggestion makes possible the elimination of a Lipschitz condition, and other simplifications. My original proof was more direct, but depended on a type of Lipschitz condition, and a modification of the method of successive approximations. The remainder of the paper is merely the result of fitting the various hypotheses together so as to make the machine work while covering as many cases as possible. A fundamental case is taken up first in $\$ \S 2$ and 3. Various generalizations are considered in $\$ 4$. Mayer problems in parametric form are considered in Part II.

A special case of the theorems of Part II, involving the existence of a minimum for a function of integrals, has been treated by Gillespie.* Other special cases of the theorems below have been given by Manià. $\dagger$ He assumes that the equations (1) have the special form

$$
z_{\sigma}^{\prime}=h_{\sigma}\left(x, y, y^{\prime}, z_{1}, \cdots, z_{\sigma}\right),
$$

where each function $h_{\sigma}$ is monotone increasing with respect to $z_{1}, \cdots, z_{\sigma-1}$, and monotone decreasing with respect to $z_{\sigma}$. The present discussion includes also systems of equations of the more general form

$$
z_{\sigma}^{\prime}=h_{\sigma}\left(x, y, y^{\prime}, z_{1}, \cdots, z_{s}\right),
$$

where each function $h_{\sigma}$ is monotone increasing with respect to each of its arguments $z_{\rho}$. As Manià has noted, certain simple types of isoperimetric problems are included as special cases. On the other hand, the more difficult isoperimetric problems in parametric form treated by Tonelli $\ddagger$ and by McShane $\S$ are not implied by the theorems of this paper.

For a systematic presentation of certain fundamental concepts used, the

* On functions of integrals, Proceedings of the Edinburgh Mathematical Society, (2), vol. 3 (1932), pp. 87-98.

† Esistenza dell'estremo assoluto in un classico problema di Mayer, Annali della Reale Scuola Normale Superiore di Pisa, (2), vol. 2 (1933), pp. 343-354; Sul problema di Mayer, Rendiconti dei Lincei, (6), vol. 18 (1933), pp. 358-365; Sui problemi di Lagrange e di Mayer, Rendiconti del Circolo Matematico di Palermo, vol. 58 (1934), pp. 285-310.

$\ddagger$ Fondamenti di Calcolo delle Variazioni, vol. II, pp. 468-482.

Semi-continuity in the calculus of variations, and absolute minima for isoperimetric problems, Dissertation, Chicago, 1930. Published in Contributions to the Calculus of Variations, 1930, Chicago, pp. 195-243. 
reader is referred to Tonelli's Fondamenti, vols. I and II, to a previous paper by the author, $\dagger$ and for the parametric problem to the dissertation by McShane already referred to.

\section{Part I. Problems in Non-Parametric Form}

1. Lower semi-continuity of solutions of differential equations. We shall use the letters $\omega, \eta, \zeta, y, z, w, h$ and their corresponding capitals as multipartite symbols, that is, $y$ shall stand for $\left(y_{1}, \cdots, y_{k}\right)$, and $z$ for $\left(z_{1}, \cdots, z_{s}\right)$. We shall also use the summation convention of tensor analysis. The subscripts $i, j$ will usually range from 1 to $k$, while $\sigma, \rho$ will usually range from 1 to $s$. The appropriate ranges for subscripts will be indicated in other cases so far as necessary by the context. The symbols $\|y\|,\|z\|$, etc., will be used to denote the maximum $\left|y_{i}\right|$, the maximum $\left|z_{\sigma}\right|$, etc. We shall be interested in certain special existence theorems for solutions of equations of the form

$$
z_{\sigma}=\zeta_{\sigma}+\int_{a}^{x} h_{\sigma}(x, z) d x .
$$

We shall suppose for Theorems 1 and 2 that the functions $h_{\sigma}(x, z)$ are defined and non-negative for $a \leqq x \leqq b$ and for all $z$, and that they are measurable on $a \leqq x \leqq b$ for fixed $z$, and continuous in $z$ for fixed $x$.

THEOREM 1. Suppose the functions $h_{\sigma}$ are motonone increasing with respect to each argument $z_{\rho}$. Then if there exists a set of bounded measurable functions $z_{\sigma}{ }^{0}(x)$ such that

$$
z_{\sigma}^{0}(x) \geqq \zeta_{\sigma}+\int_{a}^{x} h_{\sigma}\left[x, z^{0}(x)\right] d x,
$$

the equations (2) have a uniquely determined least solution $z_{\sigma}(x) \leqq z_{\sigma}{ }^{0}(x)$ on $(a, b)$.

Let

$$
z_{\sigma}^{n}(x)=\zeta_{\sigma}+\int_{a}^{x} h_{\sigma}\left(x, z^{n-1}\right) d x .
$$

Then the sequence $z_{\sigma}^{n}(x)$ is monotone decreasing with respect to $n$, and hence converges to a solution $z^{*}$ of equations (2) with $z_{\sigma}^{*}(x) \leqq z_{\sigma}{ }^{0}(x)$. If there are two solutions $z^{1}$ and $z^{2}$, let $\bar{z}$ denote the logical product of $z^{1}$ and $z^{2}$, i.e., for each $\sigma$ and $x, \bar{z}_{\sigma}(x)=$ lesser of $z_{\sigma}^{1}(x)$ and $z_{\sigma}^{2}(x)$. Then

$$
\bar{z}_{\sigma}(x) \geqq \zeta_{\sigma}+\int_{a}^{x} h_{\sigma}(x, \bar{z}) d x,
$$

† Graves, On the existence of the absolute minimum in space problems of the calculus of variations, Annals of Mathematics, vol. 28 (1927), pp. 153-170. This paper will henceforth be referred to as "Annals." 
so that by the above there is a solution $z_{\sigma}^{12}(x) \leqq \bar{z}_{\sigma}(x)$. The same reasoning is applicable to any finite number or to a denumerable infinity of solutions. Now let $z_{\sigma}{ }^{n \rho}(x)(n=1,2, \cdots ; \rho=1, \cdots, s)$ be $s$ sequences of solutions such that $\lim _{n=\infty} z_{\rho}{ }^{n \rho}(b)=B_{\rho}$, where $B_{\rho}$ is the greatest lower bound of the values $z_{\rho}(b)$ for all possible solutions $z(x)$. Then there is a solution $z(x)$ with $z_{\sigma}(x) \leqq z_{\sigma}{ }^{{ } \rho}(x)$ and hence $z_{\sigma}(b)=B_{\sigma}$. It is readily verified that there is only one solution satisfying the equations $z_{\sigma}(b)=B_{\sigma}$, and that it is the least solution of equations (2).

THEOREM 2. Suppose there is only one equation (2) and let the function $h(x, z)$ be monotone decreasing with respect to $z$. Suppose that $h(x, \zeta)$ is integrable. Then the equation (2) has a unique solution on the interval $(a, b)$.

Let $H(x, z)=h(x, z)$ for $z \geqq \zeta$, and $H(x, z)=h(x, \zeta)$ for $z<\zeta$. Then $0 \leqq H(x, z) \leqq h(x, \zeta)$, so that by a theorem of Carathéodory $\dagger$ there is a solution $z(x)$ of the equation

$$
z=\zeta+\int_{a}^{x} H(x, z) d x
$$

on the interval $(a, b)$. Since $z(x) \geqq \zeta$, this function is also a solution of equation (2). If there were two solutions $z^{1}(x)$ and $z^{2}(x)$, there would be an interval $c<x<d$ on which one solution is greater, say $z^{1}(x)>z^{2}(x)$, and such that $z^{1}(c)=z^{2}(c)$. Then

$$
z^{1}(x)=z^{1}(c)+\int_{c}^{x} h\left(x, z^{1}\right) d x \leqq z^{2}(c)+\int_{c}^{x} h\left(x, z^{2}\right) d x=z^{2}(x),
$$

which is a contradiction.

Let $A$ be a bounded closed set in $x y$-space. A curve $C$ :

$$
y_{i}=y_{i}(x) \quad(a \leqq x \leqq b)
$$

will be called admissible provided its points $(x, y(x))$ lie in $A$ and its functions $y_{i}(x)$ are absolutely continuous. The curves $C$ of a class $K$ of absolutely continuous curves are said to be equally absolutely continuous $\ddagger$ in case for every positive number $\epsilon$ there is a positive number $\delta$ such that for every curve $C$ in $K, \Sigma\left\|y\left(\alpha_{n}\right)-y\left(\beta_{n}\right)\right\|<\epsilon$ for every set of non-overlapping intervals $\left(\alpha_{n}, \beta_{n}\right)$ whose length-sum is less than $\delta$. In comparing two curves

$\dagger$ Vorlesungen über reelle Funktionen, p. 672. The existence theorem applies even when there are several equations (2), but the uniqueness apparently does not. Other theorems on the existence of maximal and minimal solutions have been given by Kamke, Acta Mathematica, vol. 58 (1932), pp. 57-85.

† See Vitali, Sull'integrazione per serie, Rendiconti del Circolo Matematico di Palermo, vol. 23 (1907), p. 139; also Annals, pp. 156-157. 
$C_{1}: y_{i}=y_{1 i}(x) \quad\left(a_{1} \leqq x \leqq b_{1}\right), \quad C_{2}: \quad y_{i}=y_{2 i}(x) \quad\left(a_{2} \leqq x \leqq b_{2}\right)$,

or two functions $z_{1 \sigma}(x)$ and $z_{2 \sigma}(x)$, it is frequently convenient to extend the range of definition of the functions involved so that they will be continuous on the whole $x$-axis, by assigning constant values to the functions outside of the original interval of definition. With this understanding, the distance $\dagger$ $\left\|C_{1}, C_{2}\right\|$ of two curves is defined to be the maximum of the quantities ||$y_{1}(x)-y_{2}(x) \|,\left|a_{1}-a_{2}\right|,\left|b_{1}-b_{2}\right|$.

Let $h_{\sigma}\left(x, y, y^{\prime}, z\right)$ be a set of functions defined and continuous together with their partial derivatives $h_{\sigma y_{i}^{\prime}}$ for $(x, y)$ in $A$ and for all $y^{\prime}$ and all $z$. An element $(C, \zeta)$ will be called admissible provided its curve

$C$ :

$$
y_{i}=y_{i}(x)
$$$$
(a \leqq x \leqq b)
$$

is admissible, and the equations

$$
z_{\sigma}(x)=\zeta_{\sigma}+\int_{a}^{x} h_{\sigma}\left[x, y(x), y^{\prime}(x), z(x)\right] d x
$$

have a continuous solution on the closed interval $(a, b)$. However, for the statement of the next two theorems it is convenient to split the $z$-variables into two groups, which will be denoted by $w$ and $z$. The corresponding initial values will be denoted by $\omega$ and $\zeta$. The $w$ 's will be supposed already determined as functions $w[x ; C, \omega]$ defined and continuous in $x$ for $(C, \omega)$ in a certain class, and for $a \leqq x \leqq b$, and the $z$ 's will be supposed to be determined as functions $z[x ; C, \omega, \zeta]$ by the equations

$$
z_{\sigma}=\zeta_{\sigma}+\int_{a}^{x} h_{\sigma}\left(x, y, y^{\prime}, w, z\right) d x .
$$

In case the functions $h_{\sigma}$ are monotone increasing with respect to their arguments $z_{\rho}$, the existence of a solution implies by Theorem 1 the existence of a uniquely determined least solution $z[x ; C, \omega, \zeta]$. The only other case we shall consider is that in which there is only one equation (3) and the function $h$ is monotone decreasing with respect to $z$. Then by Theorem 2 , there is at most one solution. The function $z_{\sigma}[x ; C, \omega, \zeta]$, regarded as defined on a class $K$ of admissible elements $(C, \omega, \zeta)$, will be said to be lower semi-continuous at an element $\left(C_{0}, \omega_{0}, \zeta_{0}\right)$ of $K$ uniformly with respect to $x$, in case for every positive number $\mu$ there is a positive number $\gamma$ such that $z_{\sigma}[x ; C, \omega, \zeta]>z_{\sigma}\left[x ; C_{0}, \omega_{0}, \zeta_{0}\right]$ $-\mu$ for all $x$ and for all $(C, \omega, \zeta)$ in $K$ such that $\left\|C, C_{0}\right\|<\gamma,\left\|\omega-\omega_{0}\right\|<\gamma$, $\left\|\zeta-\zeta_{0}\right\|<\gamma$. 
THEOREM 3. Suppose that each function $h_{\sigma}\left(x, y, y^{\prime}, w, z\right)$ is non-negative, and is monotone increasing with respect to each $w_{\tau}$ and each $z_{p}$. Suppose also that each function $h_{\sigma}$ satisfies the Weierstrass condition

$$
\begin{aligned}
E_{\sigma}\left(x, y, y^{\prime}, Y^{\prime}, z\right) \equiv & h_{\sigma}\left(x, y, Y^{\prime}, z\right)-h_{\sigma}\left(x, y, y^{\prime}, z\right) \\
& -\left(Y_{i}^{\prime}-y_{i}^{\prime}\right) h_{\sigma y_{i}^{\prime}}\left(x, y, y^{\prime}, z\right) \geqq 0
\end{aligned}
$$

for all $\left(x, y, y^{\prime}, Y^{\prime}, w, z\right)$. Let $K$ be a class of admissible elements $(C, \omega, \zeta)$ whose curves $C$ are equally absolutely continuous, and suppose that the numbers $z_{\sigma}[b ; C, \omega, \zeta]$ defined by equations (3) are bounded on $K$. Let $\left(C_{0}, \omega_{0}, \zeta_{0}\right)$ be an element of accumulation of $K$ such that the functions $w_{0 \tau}=w_{\tau}\left[x ; C_{0}, \omega_{0}\right]$ are defined and each $w_{\tau}[x ; C, \omega]$ as on $K$ is lower semi-continuous at $\left(C_{0}, \omega_{0}\right)$ uniformly with respect to $x$. Then the element $\left(C_{0}, \omega_{0}, \zeta_{0}\right)$ is admissible, and each $z_{\sigma}[x ; C, \omega$, $\zeta]$ as on $K$ is lower semi-continuous at $\left(C_{0}, \omega_{0}, \zeta_{0}\right)$ uniformly with respect to $x$.

Let $\left(C_{n}, \omega_{n}, \zeta_{n}\right)$ be an arbitrary sequence of elements of $K$ converging to $\left(C_{0}, \omega_{0}, \zeta_{0}\right)$. Since each $h_{\sigma} \geqq 0$, the corresponding functions $z_{n \sigma}(x)=z_{\sigma}\left[x ; C_{n}\right.$, $\left.\omega_{n}, \zeta_{n}\right]$ are monotone increasing functions of $x$, and since they are also uniformly bounded there exists $\dagger$ a sub-sequence which converges for every $x$ and $\sigma$ to a function $z_{\sigma}^{*}(x)$. The same notation will be used for this sub-sequence as for the original sequence. Let $z_{\sigma}^{* *}(x)$ be step functions, continuous on the right, and everywhere less than $z_{\sigma}^{*}(x)$. Then for $n$ sufficiently large, $z_{n \sigma}(x)>z_{\sigma}^{* *}(x)$ at each discontinuity of $z_{\sigma}^{* *}$ and hence for all $x$, since the functions $z_{n \sigma}$ are monotone increasing in $x$. Moreover, for every $\delta>0$ we have for $n$ sufficiently large, $w_{n \tau}(x)=w_{\tau}\left[x ; C_{n}, \omega_{n}\right] \geqq w_{0 \tau}(x)-\delta$. Hence for sufficiently large $n$,

$$
\begin{aligned}
z_{n \sigma}(x)-\zeta_{n \sigma} & =\int_{a_{n}}^{x} h_{\sigma}\left(x, y_{n}, y_{n}^{\prime}, w_{n}, z_{n}\right) d x \\
& \geqq \int_{a_{n}}^{x} h_{\sigma}\left(x, y_{n}, y_{n}^{\prime}, w_{0}-\delta, z^{* *}\right) d x .
\end{aligned}
$$

By known theorems on lower semi-continuity $\ddagger$

$$
\begin{aligned}
z_{\sigma}^{*}(x)-\zeta_{0 \sigma} & \geqq \lim \inf \int_{a_{n}}^{x} h_{\sigma}\left(x, y_{n}, y_{n}^{\prime}, w_{0}-\delta, z^{* *}\right) d x \\
& \geqq \int_{a_{0}}^{x} h_{\sigma}\left(x, y_{0}, y_{0}^{\prime}, w_{0}-\delta, z^{* *}\right) d x .
\end{aligned}
$$

† See Helly, Lineare Funktionaloperationen, Sitzungsberichte der Wiener Akademie, (IIA), vol. 121 (1912), p. 283.

$\ddagger$ See Annals, pp. 164, 165. Hypothesis (II) of Annals is not required here, since the curves of $K$ are equally absolutely continuous. 
It is easy to construct a sequence of step functions $z^{* *}$ converging to $z^{*}$ except at the discontinuities of the latter. Since $\delta$ is also arbitrary, we have finally

$$
z_{\sigma}^{*}-\zeta_{0 \sigma} \geqq \int_{a_{0}}^{x} h_{\sigma}\left(x, y_{0}, y_{0}^{\prime}, w_{0}, z^{*}\right) d x,
$$

and the proof implies the existence of the integral. From Theorem 1 it follows that the element $\left(C_{0}, \omega_{0}, \zeta_{0}\right)$ is admissible, and $z_{0 \sigma}(x) \equiv z_{\sigma}\left[x ; C_{0}, \omega_{0}, \zeta_{0}\right]$ $\leqq z_{\sigma}^{*}(x)=\lim z_{n \sigma}(x)$. Suppose now that the final statement of the theorem is not true. Then there exists a positive constant $\mu$, a value of the index $\sigma$, and sequences $\left(C_{n}, \omega_{n}, \zeta_{n}\right)$ and $\left(x_{n}\right)$, such that $\left(C_{n}, \omega_{n}, \zeta_{n}\right)$ converges to $\left(C_{0}, \omega_{0}, \zeta_{0}\right)$, and $z_{n \sigma}\left(x_{n}\right)<z_{0 \sigma}\left(x_{n}\right)-\mu$. These sequences may be chosen as in the preceding part of the proof and also so that $x_{n}$ converges to a point $\bar{x}$. Choose $\alpha$ so small that $z_{0 \sigma}(\bar{x}-\alpha)>z_{0 \sigma}(\bar{x})-\mu / 3$. Then for $n$ sufficiently large, $z_{n \sigma}\left(x_{n}\right)$ $\geqq z_{n \sigma}(\bar{x}-\alpha) \geqq z_{0 \sigma}(\bar{x}-\alpha)-\mu / 3, \quad z_{0 \sigma}(\bar{x})>z_{0 \sigma}\left(x_{n}\right)-\mu / 3$, and these inequalities with the two preceding readily yield a contradiction.

THEOREM 4. Suppose the hypotheses of Theorem 3 hold, except that there is only one $z$ and one $h$, and the function $h$ is monotone decreasing with respect to $z$. Let the function $h$ satisfy the condition

(L) for every admissible curve $C$ and set of continuous functions $w_{\tau}(x), z^{1}(x)$, $z^{2}(x)$, for which $h\left[x, y(x), y^{\prime}(x), w(x), z^{1}(x)\right]$ is integrable, the function $h\left[x, y(x), y^{\prime}(x), w(x), z^{2}(x)\right]$ is also integrable. $\dagger$

Then the conclusions of Theorem 3 are still valid. $\ddagger$

As before, we consider a sub-sequence $\left(C_{n}, \omega_{n}, \zeta_{n}\right)$ of an arbitrary sequence of elements of $K$ converging to $\left(C_{0}, \omega_{0}, \zeta_{0}\right)$, such that $z_{n}(x)=z\left[x ; C_{n}, \omega_{n}, \zeta_{n}\right]$ converges to a function $z^{*}(x)$. Let $z^{* *}(x)$ be a step function, continuous on the left, and everywhere greater than $z^{*}(x)$, and let $\alpha$ and $\beta$ be such that $a_{0} \leqq \alpha<\beta \leqq b_{0}$. Then for $n$ sufficiently large,

$$
z_{n}(\beta)-z_{n}(\alpha)=\int_{\alpha}^{\beta} h\left(x, y_{n}, y_{n}^{\prime}, w_{n}, z_{n}\right) d x
$$

$\dagger$ This condition is implied by a suitable Lipschitz condition, or by the condition $(\tau)$ of Manià. See Rendiconti del Circolo Matematico di Palermo, loc. cit., pp. 303, 307.

$\ddagger$ The following examples, in which the solutions of the differential equations fail to be lower semi-continuous, illustrate the need for the hypothesis that the function $h$ shall be monotone increasing with respect to the variables $w$. It will be noticed that the hypotheses (III), (V) to (VIII) of the existence theorem of $\S 2$ are all fulfilled. Let $w^{\prime}=-w\left(1+y_{1}^{\prime 2}+y_{2}^{\prime 2}\right)^{1 / 2}+y_{1}^{\prime 2}+y_{2}^{\prime 2}, z^{\prime}=-w$ for $w \leqq 0$, and $w^{\prime}=y_{1}^{\prime 2}+y_{2}^{\prime 2}, z^{\prime}=0$ for $w>0$. Let the initial values be $\omega=-2, \zeta=0$, for $x=0$. Then $z$ is not lower semi-continuous at the curve $C_{0}: y_{1}=0, y_{2}=0$ for $x$ small, as may be seen by considering the sequence $C_{n}: y_{1}=(\cos n x) / n, y_{2}=(\sin n x) / n$. Another example is the following: $w^{\prime}=e^{-w}\left(1+y_{1}^{\prime 2}\right.$ $\left.+y_{2}^{\prime 2}\right)^{1 / 2}+y_{1}^{\prime 2}+y_{2}^{\prime 2}, z^{\prime}=e^{-w}$, with initial conditions $\omega=\zeta=0$ for $x=0$. 


$$
\geqq \int_{\alpha}^{\beta} h\left(x, y_{n}, y_{n}^{\prime}, w_{0}-\delta, z^{* *}\right) d x,
$$

where it is agreed that the integrand $h$ is to be replaced by zero at points of the interval $(\alpha, \beta)$, if any, falling outside the interval $\left(a_{n}, b_{n}\right)$. By the same reasoning as before we find that

$$
z^{*}(\beta)-z^{*}(\alpha) \geqq \int_{\alpha}^{\beta} h\left(x, y_{0}, y_{0}^{\prime}, w_{0}, z^{*}\right) d x .
$$

From the condition (L) it follows that $h\left(x, y_{0}, y_{0}^{\prime}, w_{0}, \zeta_{0}\right)$ is integrable, and by Theorem 2, $\left(C_{0}, \omega_{0}, \zeta_{0}\right)$ is admissible. Suppose now that $z^{*}(\beta)<z_{0}(\beta)$. Then since $z_{0}(x)=z\left[x ; C_{0}, \omega_{0}, \zeta_{0}\right]$ is monotone increasing and continuous, and $z^{*}(x)$ is monotone increasing, there is a last point $\alpha<\beta$ at which $z^{*}(\alpha)=z_{0}(\alpha)$. On the interval $\alpha<x \leqq \beta, z^{*}(z)<z_{0}(x)$, and hence

$$
\begin{aligned}
\int_{\alpha}^{\beta} h\left(x, y_{0}, y_{0}^{\prime}, w_{0}, z_{0}\right) d x & \leqq \int_{\alpha}^{\beta} h\left(x, y_{0}, y_{0}^{\prime}, w_{0}, z^{*}\right) d x \\
& \leqq z^{*}(\beta)-z^{*}(\alpha)<z_{0}(\beta)-z_{0}(\alpha) .
\end{aligned}
$$

This is a contradiction. Now from the result $z_{0}(x) \leqq z^{*}(x)$ we may argue the uniform lower semi-continuity as before.

2. A first existence theorem. We shall be concerned with functions $g(a, b, \eta, Y, \zeta, Z)$ and $h_{\sigma}\left(x, y, y^{\prime}, z\right)$ satisfying the following hypotheses.

(I) $g(a, b, \eta, Y, \zeta, Z)$ is defined and continuous for $(b, Y)$ in the bounded closed set $A,(a, \eta, \zeta)$ in a bounded closed set $S$ in $(x, y, z)$-space, and all $Z$, and is monotone increasing with respect to each argument $Z_{\sigma}$.

(II) There is a non-null subset of $[1 \leqq \sigma \leqq s]$, denoted by $\left[\sigma^{*}\right]$, such that $g(a, b, \eta, Y, \zeta, Z) \rightarrow+\infty$ with each $Z_{\sigma^{*}}$, the remaining arguments being fixed.

(III) The functions $h_{\sigma}\left(x, y, y^{\prime}, z\right)$ and their partial derivatives with respect to the $y_{i}^{\prime}$ are defined and continuous for $(x, y)$ in $A$ and for all $y^{\prime}$ and $z$. For the sake of greater generality in the following hypotheses we suppose that the range $[1 \leqq \sigma \leqq s]$ is divided into subsets by integers $0=r_{0}<r_{1}<r_{2}<\cdots$, in such a way that each $h_{\sigma}$ having $\sigma \leqq r_{k}$ is independent of all the $z_{\rho}$ having $\rho>r_{k}$. It is understood of course that we may have $r_{1}=s$.

(IV) Each function $h_{\sigma}$ is either (a) monotone increasing with respect to each argument $z_{\rho}$; or (b) monotone increasing with respect to each $z_{\rho}$ for $\rho<\sigma$ and monotone decreasing with respect to $z_{\sigma}$, and $\sigma=r_{j}=1+r_{j-1}$, i.e., $\sigma$ belongs to a subset consisting of only one element. 
(V) Each function $h_{\sigma}$ which is monotone decreasing with respect to $z_{\sigma}$ satisfies the condition (L) stated in Theorem 4, with the functions $w_{\tau}(x)$ replaced by $z_{1}(x), \cdots, z_{\sigma-1}(x)$.

(VI) The functions $h_{\sigma}$ satisfy the Weierstrass condition, i.e., $E_{\sigma}\left(x, y, y^{\prime}, Y^{\prime}\right.$, $z) \geqq 0$ for $(x, y)$ in $A$, and all $y^{\prime}, Y^{\prime}, z$.

(VII) The functions $h_{\sigma}$ are non-negative for $(x, y)$ in $A$ and all $y^{\prime}, z$.

(VIII) At least one of the functions $h_{\sigma}$ satisfies the condition that for every positive number $N$ there exists a constant $M$ and a function $\Phi(u)$ such that (1) $\Phi$ is positive, continuous, and monotone increasing for $u \geqq 0$; (2) $\lim _{u=+\infty} \Phi(u) / u=+\infty$; (3) $h_{\sigma}\left(x, y, y^{\prime}, z\right) \geqq \Phi\left(\left\|y^{\prime}\right\|\right)$ for all $(x, y)$ in $A$, and $\|z\| \leqq N,\left\|y^{\prime}\right\|>M$.

(IX) For every $\sigma$ not in the class $\left[\sigma^{*}\right]$ there exist a $\sigma^{*}$, a positive constant $\mu$, and an integrable function $\nu(x)$ such that $h_{\sigma}\left(x, y, y^{\prime}, z\right) \leqq \mu h_{\sigma^{*}}\left(x, y, y^{\prime}, z\right)+\nu(x)$ for all $(x, y)$ in $A$ and all $y^{\prime}, z$.

Admissible elements $(C, \zeta)$ have been defined in $\S 1$. A class $K$ of admissible elements $(C, \zeta)$ is said to be closed in case it contains all its elements of accumulation $\left(C_{0}, \zeta_{0}\right)$ which are admissible. For example, a class consisting of all admissible elements $(C, \zeta)$ whose end values satisfy equations of the form $\psi_{\mu}[a, b, y(a), y(b), \zeta]=0$ is a closed class, provided the $\psi_{\mu}$ are continuous. A class $K$ will be called quasi-closed in case it contains all elements $\left(C_{0}, \zeta_{0}\right)$ which are admissible and are limits of sequences $\left\{C_{n}, \zeta_{n}\right\}$ chosen from $K$, whose curves $C_{n}$ are equally absolutely continuous. The existence theorems will be stated for quasi-closed classes, since they are then more generally applicable. We note that if $K$ is a class of admissible elements $(C, \zeta)$ having initial values $(a, y(a), \zeta)$ in the set $S$, then the function

$$
I(C, \zeta)=g(a, b, y(a), y(b), \zeta, z[b ; C, \zeta])
$$

is well defined on $K$.

Existence Theorem. Let the functions $g$ and $h_{\sigma}$ satisfy the preceding hypotheses (I)-(IX), and let $K$ be a non-null bounded quasi-closed class of admissible elements $(C, \zeta)$ having initial values $(a, y(a), \zeta)$ in the set $S$. Then $I(C, \zeta)$ has a minimum on $K$.

3. Proof of the existence theorem. We first prove two lemmas.

LEMMA 1. Let $K_{1}$ be a class of admissible elements $(C, \zeta)$ on which the functions $z_{\sigma}[x ; C, \zeta]$ are all bounded. Then the curves of $K_{1}$ are equally absolutely continuous. 
Let $h_{\sigma_{0}}$ be a function satisfying hypothesis (VIII). Let $N \geqq\|z[x ; C, \zeta]\|$ and $B \geqq z_{\sigma_{0}}[b ; C, \zeta]-\zeta_{\sigma_{0}}$ on $K_{1}$, and let $E_{1} \equiv E\left[\left\|y^{\prime}\right\|>M\right], E_{2} \equiv E\left[\left\|y^{\prime}\right\| \leqq M\right]$, $D \equiv$ maximum difference of abscissas of points in $A$. Then

$$
\begin{aligned}
\int_{a}^{b} \Phi\left(\left\|y^{\prime}\right\|\right) d x & \leqq \int_{E_{1}} h_{\sigma_{0}}\left(x, y, y^{\prime}, z\right) d x+\int_{E_{2}} \Phi(M) d x \\
& \leqq B+D \Phi(M) .
\end{aligned}
$$

From this inequality the equal absolute continuity of the curves of $K_{1}$ readily follows. $\dagger$

LEMMA 2. Let $K_{1}$ be a class of admissible elements $(C, \zeta)$ having initial values $(a, y(a), \zeta)$ in the set $S$, and let $I(C, \zeta)$ be bounded above on $K_{1}$. Then each function $z_{\sigma}[b ; C, \zeta]$ is bounded on $K_{1}$.

Suppose the lemma is false, and let $\left\{C_{n}, \zeta_{n}\right\}$ be a sequence selected from $K_{1}$, such that the corresponding sequence

$$
\left\{a_{n}, b_{n}, y_{n}\left(a_{n}\right), y_{n}\left(b_{n}\right), \zeta_{n}, Z_{n}\right\} \rightarrow\left(a_{0}, b_{0}, \eta_{0}, Y_{0}, \zeta_{0}, Z_{0}\right),
$$

where $Z_{n}=z\left[b_{n} ; C_{n}, \zeta_{n}\right]$, and at least one component $Z_{0 \sigma}=+\infty$. By hypothesis (IX) there must be a $\sigma^{*}$ such that $Z_{0 \sigma^{*}}=+\infty$. The sequences $Z_{n \sigma}$ are bounded below, say $\geqq B_{\sigma}$, since the set $S$ is bounded and each $h_{\sigma} \geqq 0$. Let $Z_{n \sigma}^{*}=B_{\sigma}$ for $\sigma$ different from the particular $\sigma^{*}$ just mentioned, and $Z_{n \sigma^{*}}^{*}=Z_{n \sigma^{*}}$. Then for each $n$ there is an $m>n$ such that when $q>m$,

$$
g\left[a_{q}, b_{q}, y_{q}\left(a_{q}\right), y_{q}\left(b_{q}\right), \zeta_{q}, Z_{n}^{*}\right] \leqq g\left[a_{q}, b_{q}, y_{q}\left(a_{q}\right), y_{q}\left(b_{q}\right), \zeta_{q}, Z_{q}\right\rfloor=I\left(C_{q}, \zeta_{q}\right),
$$

which is bounded. Hence $g\left[a_{0}, b_{0}, \eta_{0}, Y_{0}, \zeta_{0}, Z_{n}^{*}\right]$ is bounded, which contradicts hypothesis (II).

To complete the proof of the theorem, let $i$ be the greatest lower bound of $I(C, \zeta)$ on the class $K$. It is easily seen that $i$ is finite. If $\left\{C_{n}, \zeta_{n}\right\}$ is a minimizing sequence, it follows from Lemmas 2 and 1 that each component $z_{\sigma}\left[b_{n} ; C_{n}, \zeta_{n}\right]$ is bounded and that the curves $C_{n}$ are equally absolutely continuous. From this we know by applying the theorems of Ascoli and Weierstrass-Bolzano that the sequence $\left\{C_{n}, \zeta_{n}\right\}$ has an element of accumulation $\left(C_{0}, \zeta_{0}\right)$. The curve $C_{0}$ is absolutely continuous and lies in $A$, while the initial values $\left(a_{0}, y_{0}\left(a_{0}\right), \zeta_{0}\right)$ lie in the set $S$. From Theorems 3 and 4 it follows that the element $\left(C_{0}, \zeta_{0}\right)$ is admissible and hence in the class $K$, and that

$$
\lim \inf z_{\sigma}\left[b_{n} ; C_{n}, \zeta_{n}\right] \geqq z_{\sigma}\left[b_{0} ; C_{0}, \zeta_{0}\right] .
$$

From the properties assumed for the function $g$ it follows without difficulty that $i=\lim I\left(C_{n}, \zeta_{n}\right) \geqq I\left(C_{0}, \zeta_{0}\right) \geqq i$.

† See Nagumo, Ueber die gleichmaessige Summierbarkeit und ihre Anwendung auf ein Variationsproblem, Japanese Journal of Mathematics, vol. 6 (1929), p. 173. 
4. Generalizations. The existence theorem stated in $\$ 2$ may be generalized in several ways. Let us consider first certain restrictions on the range of $y^{\prime}$. Let $\psi_{p i}, \Psi_{p}, \theta_{q i}, \Theta_{q}, \phi_{r}$ be functions of $x$ and $y_{i}$ which, together with the partial derivatives $\phi_{r x}, \phi_{r y_{j}}$, are defined and continuous on the set $A$. Let $R$ denote the set of $\left(x, y, y^{\prime}\right)$ points having $(x, y)$ in $A$ and satisfying the conditions

$$
\begin{aligned}
& \phi_{r}=0, \quad \phi_{r y_{i}} y_{i}^{\prime}+\phi_{r x}=0, \\
& \psi_{p i} y_{i}^{\prime}+\Psi_{p}=0, \quad \theta_{q i} y_{i}^{\prime}+\Theta_{q} \geqq 0 .
\end{aligned}
$$

A curve $C: y_{i}=y_{i}(x)$ will be said to lie in $R$ in case its elements $\left(x, y(x), y^{\prime}(x)\right)$ lie in $R$ for almost all $x$. The hypotheses (III)-(IX) inclusive may be modified by assuming that they hold only for $\left(x, y, y^{\prime}\right)$ in $R$, except that (VI) has the form $E_{\sigma}\left(x, y, y^{\prime}, Y^{\prime}, z\right) \geqq 0$ for every $\left(x, y, Y^{\prime}\right)$ and $\left(x, \bar{y}, y^{\prime}\right)$ in $R$ and every $z$. $\dagger$ Let the class $K$ of the existence theorem satisfy the additional condition that its curves $C$ lie in $R$. Then $I(C, \zeta)$ has a minimum on $K$. The proof is essentially the same as before.

It is important to note that if $K_{0}$ is a given quasi-closed class of admissible elements $(C, \zeta)$, and $K$ consists of all the elements of $K_{0}$ whose curves $C$ lie in $R$, then $K$ is also quasi-closed. This is a consequence of the

LEMMA 3. Let $\left\{C_{n}\right\}$ be a sequence of curves in $R$, equally absolutely continuous, and converging to a curve $C_{0}$. Then $C_{0}$ also lies in $R$.

It is clear that the equations $\phi_{r}(x, y)=0$ will be satisfied by $C_{0}$. Consider now the function $w$ defined by an equation of the form

$$
w[x ; C]=\int_{a}^{x}\left\{\psi_{i}(x, y) y_{i}^{\prime}+\Psi(x, y)\right\} d x .
$$

Since the Weierstrassian $E$-function vanishes identically for an integrand linear in $y^{\prime}$, it follows from a theorem on semi-coníinuity previously quoted that $\lim w\left[x ; C_{n}\right]=w\left[x ; C_{0}\right]$. If each $w\left[x ; C_{n}\right]$ vanishes identically, so does $w\left[x ; C_{0}\right]$. If each $w\left[x ; C_{n}\right]$ is a monotone increasing function of $x$, so is $w\left[x ; C_{0}\right]$. This argument shows at once that $C_{0}$ lies in $R$.

As a second generalization we may impose certain restrictions on the range of $z_{\sigma}$ and $Z_{\sigma}$. Let $\beta_{\sigma}(x, y)$ be functions defined on the set $A$, with values which are finite or $-\infty$, bounded above, and continuous except for infinite discontinuities. The subset of $A$ on which a given $\beta_{\sigma}=-\infty$ is then unre stricted except that it is closed. Let $B_{\sigma}(x, y)$ be similar functions, with values finite or $+\infty$, and bounded below. The hypotheses are modified as follows:

† See Graves, Bulletin of the American Mathematical Society, loc. cit. In certain cases this hypothesis may be weakened by requiring $\bar{y}=y$. A modification of the proof is required. 
(I) holds only for $\beta_{\sigma}(b, y) \leqq Z_{\sigma} \leqq B_{\sigma}(b, Y)$, and the initial values in the set $S$ satisfy $\ddagger \beta_{\sigma}(a, \eta) \leqq \zeta_{\sigma} \leqq B_{\sigma}(a, \eta)$. (II) has no meaning and is omitted for those values of $\sigma^{*}, b, Y$ for which $B_{\sigma^{*}}(b, Y)$ is finite. The remaining hypotheses (III)-(IX) are understood to hold only for $\beta_{\sigma}(x, y) \leqq z_{\sigma} \leqq B_{\sigma}(x, y)$. It is easily seen that the range of the functions $h_{\sigma}$ may be extended to the whole $z$-space by setting $h_{\sigma}\left(x, y, y^{\prime}, z\right)=h_{\sigma}\left(x, y, y^{\prime}, \bar{z}\right)$, where $\bar{z}_{\rho}=\beta_{\rho}(x, y)$ if $z_{\rho}<\beta_{\rho}(x, y)$, and $\bar{z}_{\rho}=B_{\rho}(x, y)$ if $z_{\rho}>B_{\rho}(x, y)$. The original hypotheses (III)-(IX) are satisfied by the functions $h_{\sigma}$ so extended. With reference to the extended functions $h_{\sigma}$ the term "admissible element $(C, \zeta)$ " will be used in the same sense as before.

Let $K_{0}$ be a quasi-closed class of admissible elements, and let $K$ be the sub-class of $K_{0}$ consisting of all those elements $(C, \zeta)$ having initial values $(a, y(a), \zeta)$ in the set $S, \zeta_{\sigma} \geqq$ maximum of $\beta_{\sigma}(x, y(x))$, and $z_{\sigma}[x ; C, \zeta]$ $\leqq B_{\sigma}(x, y(x))$. Then from Theorems 3 and 4 it follows that $K$ will be quasiclosed. The existence theorem is evidently valid for quasi-closed classes $K$ of elements satisfying the conditions just written down.

As a third generalization we note that certain of the hypotheses may be weakened as follows. In place of (VIII) we may assume that there exists a function $f(x, y)$ of class $C^{\prime}$ on the set $A$ such that one of the functions $\bar{h}_{\sigma}\left(x, y, y^{\prime}, z\right)=h_{\sigma}\left(x, y, y^{\prime}, z\right)+f_{x}(x, y)+f_{y_{i}}(x, y) y_{i}^{\prime}$ satisfies (VIII). Condition (VIII) enters only in the proof of Lemma 1, which we revise as follows;

$$
\begin{aligned}
\int_{a}^{b} \Phi\left(\left\|y^{\prime}\right\|\right) d x \leqq & \int_{E_{1}} \bar{h}_{\sigma_{0}} d x+\int_{E_{2}} \Phi(M) d x \\
= & \int_{E_{1}} h_{\sigma_{0}} d x+\int_{E_{2}} \Phi(M) d x+f(b, y(b)) \\
& -f(a, y(a))-\int_{E_{2}}\left[f_{x}+f_{y_{i}} y_{i}^{\prime}\right] d x .
\end{aligned}
$$

Since $\left\|y^{\prime}\right\|$ is uniformly bounded on the set $E_{2}$, the additional terms are easily seen to be bounded.

In place of (VII) we may assume that there exist functions $F_{\sigma}(x, y)$, of class $C^{\prime}$ on $A$, such that the functions $h_{\sigma} \dagger\left(x, y, y^{\prime}, z \dagger\right)=h_{\sigma}\left(x, y, y^{\prime}, z \dagger-F(x, y)\right)$ $+F_{\sigma x}(x, y)+F_{\sigma y_{i}}(x, y) y_{i}^{\prime}$ satisfy condition (VII). If we use also the transformation $z_{\sigma} \dagger=z_{\sigma}+F_{\sigma}(x, y), \zeta_{\sigma} \dagger=\zeta_{\sigma}+F_{\sigma}(a, y(a)), g \dagger(a, b, \eta, Y, \zeta \dagger, Z \dagger)=g(a, b, \eta, Y, \zeta \dagger$ $-F(a, \eta), Z \dagger-F(b, Y))$, we see that the transformed functions still satisfy the hypotheses (I)-(VI) and the weakened form of (VIII). In case there are restrictions $\beta_{\sigma}(x, y) \leqq z_{\sigma} \leqq B_{\sigma}(x, y)$ on the $z_{\sigma}$, we note that the transformed re-

$\ddagger$ Whenever a $\beta_{\sigma}$ or $B_{\sigma}$ is infinite, the corresponding inequality is to be omitted. 
gions $\beta_{\sigma}(x, y)+F_{\sigma}(x, y) \leqq z_{\sigma} \dagger \leqq B_{\sigma}(x, y)+F_{\sigma}(x, y)$ have all the properties required of the original ones.

Condition (IX) may be modified in the same way as (VII), but the functions $F_{\sigma}(x, y)$ need not be the same, since (IX) enters only in the proof of Lemma 2, and the transformed functions $z_{\sigma} \dagger$ are bounded if and only if the $z_{\sigma}$ are bounded.

Finally, the restriction that the set $A$ of $(x, y)$ points is bounded may be removed in the usual way. Hypotheses (VIII) and (IX) are assumed to hold only for every bounded subset $A_{1}$ of $A$. Hypotheses (I)-(VII) require no change. It is assumed that the end values $(a, \eta, \zeta)$ and $(b, Y)$ of elements $(C, \zeta)$ of the class $K$ in which a minimum is sought lie in bounded closed sets. We require in addition the condition

(X) There is a value $\sigma^{*}$ of the index $\sigma$, belonging to the class mentioned in hypothesis (II), such that $z_{\sigma^{*}}[b ; C, \zeta] \rightarrow+\infty$ with the maximum distance of a point of $C$ from the origin in $(x, y)$-space.

Under these hypotheses, Lemma 2 still holds and its proof implies also that the curves $C$ involved lie in a bounded subset of the set $A$.

The condition (X) is implied by various conditions more directly applicable to the functions $h_{\sigma}$, as has been indicated for simpler problems by Tonelli and by Graves. $\$$

\section{Part II. Problems in parametric form}

5. Lower semi-continuity of solutions of differential equations. The theory for problems in parametric form involves only the usual modifications of the preceding. We let $A$ denote a bounded closed set in the $k$-dimensional $y$-space. A curve

$C:$

$$
y_{i}=y_{i}(t)
$$$$
(a \leqq t \leqq b)
$$

is admissible provided it is rectifiable and lies in $A$. We shall always suppose the parameter $t$ so chosen that the functions $y_{i}(t)$ are absolutely continuous. Let the functions $h_{\sigma}\left(y, y^{\prime}, z\right)$ together with their partial derivatives $h_{\sigma y_{i}^{\prime}}$, be defined and continuous for $y$ in $A$, all $y^{\prime} \neq 0$, and all $z$, and let each $h_{\sigma}\left(y, y^{\prime}, z\right)$ be positively homogeneous of degree one in the arguments $y_{i}^{\prime}$. An element $(C, \zeta)$ is admissible provided $C$ is admissible and the equations

$$
z_{\sigma}(t)=\zeta_{\sigma}+\int_{a}^{t} h_{\sigma}\left(y, y^{\prime}, z\right) d t
$$

$\ddagger$ Fondamenti, vol. II, pp. 308, 311.

$\S$ Annals, p. 168; Bulletin of the American Mathematical Society, loc. cit. 
have a continuous solution $z_{\sigma}[t ; C, \zeta]$ on $a \leqq t \leqq b$. On account of the homogeneity assumed for the functions $h_{\sigma}$, solutions of these equations are invariant under change of parameter so long as the functions $y_{i}(t)$ remain absolutely continuous. As before, we shall suppose for the next two theorems that the functions $h_{\sigma}$ depend also on certain variables $w_{r}$, and that the $w$ 's are already determined as functions $w_{\tau}[t ; C, \omega]$ of $C$ and initial values $\omega$. The function $z_{\sigma}[t ; C, \omega, \zeta]$, regarded as defined on a class $K$ of admissible elements $(C, \omega, \zeta)$, will be said to be uniformly lower semi-continuous at an element $\left(C_{0}, \omega_{0}, \zeta_{0}\right)$ of $K$ in case for every positive number $\mu$ there is a positive number $\gamma$ such that, for every element $(C, \omega, \zeta)$ in $K$ and choice of parameter such that

$$
\left\|\omega-\omega_{0}\right\|<\gamma,\left\|\zeta-\zeta_{0}\right\|<\gamma,\left\|y(t)-y_{0}(t)\right\|<\gamma \quad(a \leqq t \leqq b),
$$

we have $z_{\sigma}[t ; C, \omega, \zeta]>z_{\sigma}\left[t ; C_{0}, \omega_{0}, \zeta_{0}\right]-\mu(a \leqq t \leqq b)$.

THEOREM 5. Suppose that each function $h_{\sigma}\left(y, y^{\prime}, w, z\right)$ is non-negative, and is monotone increasing with respect to each $w_{\tau}$ and each $z_{\rho}$. Suppose also that each function $h_{\sigma}$ satisfies the Weierstrass condition $E_{\sigma}\left(y, y^{\prime}, Y^{\prime}, w, z\right)=h_{\sigma}\left(y, Y^{\prime}, w, z\right)$ $-Y_{i}^{\prime} h_{\sigma y_{i}^{\prime}}\left(y, y^{\prime}, w, z\right) \geqq 0$ for all $y$ in $A, y^{\prime} \neq 0, Y^{\prime} \neq 0$, and all w and $z$. Let $K$ be a class of admissible elements $(C, \omega, \zeta)$ whose curves $C$ have bounded lengths, and suppose that the numbers $z_{\sigma}[b ; C, \omega, \zeta]$ defined by equations (4) are bounded on $K$. Let $\left(C_{0}, \omega_{0}, \zeta_{0}\right)$ be an element of accumulation of $K$ such that the functions $w_{0 \tau}=w_{\tau}\left[t ; C_{0}, \omega_{0}\right]$ are defined and each $w_{\tau}[t ; C, \omega]$ as on $K$ is uniformly lower semi-continuous at $\left(C_{0}, \omega_{0}\right)$. Then the element $\left(C_{0}, \omega_{0}, \zeta_{0}\right)$ is admissible, and each $z_{\sigma}[t ; C, \omega, \zeta]$ as on $K$ is uniformly lower semi-continuous at $\left(C_{0}, \omega_{0}, \zeta_{0}\right)$.

Let $\left(C_{n}, \omega_{n}, \zeta_{n}\right)$ be an arbitrary sequence of elements of $K$, converging to $\left(C_{0}, \omega_{0}, \zeta_{0}\right)$. We may choose an arbitrary fixed representation of $C_{0}: y_{i}=y_{0 i}(t)$, and then select the representation of $C_{n}: y_{i}=y_{n i}(t)$ in any manner such that the functions $y_{n i}(t)$ converge uniformly to $y_{0 i}(t)$. We proceed as in the proof of Theorem 3 to show that the element $\left(C_{0}, \omega_{0}, \zeta_{0}\right)$ is admissible and that for a properly chosen sub-sequence, $\lim z_{\sigma}\left[t ; C_{n}, \omega_{n}, \zeta_{n}\right] \geqq z_{\sigma}\left[t ; C_{0}, \omega_{0}, \zeta_{0}\right]$. Suppose that the uniform lower semi-continuity at $\left(C_{0}, \omega_{0}, \zeta_{0}\right)$ is false. Then there exist a positive number $\mu$, a value of the index $\sigma$, and a sequence of elements $\left(C_{n}, \omega_{n}, \zeta_{n}\right)$ converging to $\left(C_{0}, \omega_{0}, \zeta_{0}\right)$ with representations $C_{n}: y_{i}=y_{n i}(t)$, $C_{0}: y_{i}=y_{0 i}^{n}(t)$, such that $\left\|y_{n}(t)-y_{0}{ }^{n}(t)\right\| \rightarrow 0$ uniformly, while for a properly. selected value $t_{n}$ of the parameter, $z_{n \sigma}\left(t_{n}\right) \leqq z_{0 \sigma}^{n}\left(t_{n}\right)-\mu$. Let $y_{i}=y_{0 i}(s)$ be the representation of $C_{0}$ in terms of its arc length as parameter, and let the functions $z_{0 \sigma}(s)$ correspond to this representation. Then by means of functions

$\ddagger$ For the lower semi-continuity of $\int_{a}^{t} h_{\sigma}\left(y, y^{\prime}, w_{0}-\delta, z^{* *}\right) d t$, see McShane, Dissertation, p. 11, Theorem I. 
$t=\phi_{n}(s)$ we may transform the parameter on the curves $C_{n}$ so that for the new representation $y_{i}=\bar{y}_{n i}(s)$ we still have $\left\|\bar{y}_{n}(s)-y_{0}(s)\right\| \rightarrow 0$ uniformly. To the values $t_{n}$ of the parameter there correspond values $s_{n}$ and $\bar{s}_{n}$ such that $\bar{z}_{n \sigma}\left(\bar{s}_{n}\right) \leqq z_{0 \sigma}\left(s_{n}\right)-\mu$. It is easily verified that $s_{n}-\bar{s}_{n} \rightarrow 0$. From this and from the preceding part of the proof it follows that the sequence $\left\{C_{n}, \omega_{n}, \zeta_{n}\right\}$ and a value $s_{0}$ may be so chosen that $s_{n} \rightarrow s_{0}, \bar{s}_{n} \rightarrow s_{0}$, and $\lim \bar{z}_{n \sigma}\left(s_{0}-\alpha\right) \geqq z_{0 \sigma}\left(s_{0}-\alpha\right)$, where $\alpha$ is an arbitrary positive number. Then for $\alpha$ sufficiently small and $n$ sufficiently large, $z_{0 \sigma}\left(s_{0}-\alpha\right)>z_{0 \sigma}\left(s_{n}\right)-\mu / 2, \bar{z}_{n \sigma}\left(\bar{s}_{n}\right) \geqq \bar{z}_{n \sigma}\left(s_{0}-\alpha\right)$, and from these inequalities and the preceding we readily obtain a contradiction.

THEOREM 6. Let the hypotheses of Theorem 5 hold, except that there is only one $z$ and one $h$, and the function $h$ is monotone decreasing with respect to $z$. Then the conclusions of Theorem 5 are still valid.

In this case the element $\left(C_{0}, \omega_{0}, \zeta_{0}\right)$ is seen at once to be admissible, by virtue of Theorem 2, since the function $h\left(y_{0}, y_{0}^{\prime}, w_{0}, \zeta_{0}\right)$ is bounded when the arc length is chosen as parameter. The remainder of the proof may be made as for Theorems 4 and 5 .

6. An existence theorem. For the parametric problem certain of the hypotheses made in $\$ 2$ are modified as follows.

(I) The arguments $a$ and $b$ are omitted from $g$.

(III) The functions $h_{\sigma}$ are independent of $x$, and are positively homogeneous of degree one in $y^{\prime}$. The partial derivatives $h_{\sigma y_{i}^{\prime}}$ are not defined for $y^{\prime}=0$.

$(\mathrm{V})$ is omitted.

(VIII) At least one function $h_{\sigma}$ satisfies the condition that for every positive number $N$ there exists a positive constant $m$ such that $h_{\sigma}\left(y, y^{\prime}, z\right) \geqq m$ for all $y$ in $A,\left\|y^{\prime}\right\|=1$, and $\|z\| \leqq N$.

(IX) For every $\sigma$ not in the class $\left[\sigma^{*}\right]$ there exist a $\sigma^{*}$ and a positive constant $\mu$ such that $h_{\sigma}\left(y, y^{\prime}, z\right) \leqq \mu h_{\sigma^{*}}\left(y, y^{\prime}, z\right)$ for all $y$ in $A$, all $y^{\prime}$ and all $z$.

A quasi-closed class of admissible elements $(C, \zeta)$ is now defined to be a class $K$ which contains all elements $\left(C_{0}, \zeta_{0}\right)$ which are admissible and are limits of sequences $\left\{C_{n}, \zeta_{n}\right\}$ chosen from $K$ whose curves $C_{n}$ have uniformly bounded lengths.

EXISTENCE ThEOREM. Let the functions $g$ and $h_{\sigma}$ satisfy the hypotheses (I)(IV) and (VI)-(IX) as modified, and let $K$ be a non-null bounded quasi-closed class of admissible elements $(C, \zeta)$ having initial values in the set $S$. Then $I(C, \zeta)$ has a minimum on $K$.

The proof follows the same lines as before, except for the obvious modifi- 
cation in Lemma 1. Moreover, the theorem admits of the same generalizations as those described in $\$ 4$ for the non-parametric problem. There is one deviation in the generalized hypotheses, in that when there are differential equations $\psi_{p i}(y) y_{i}^{\prime}=0$, etc., linear in the $y_{i}^{\prime}$, the condition (VI) now has the form $E_{\sigma}\left(y, y^{\prime}, Y^{\prime}, z\right) \geqq 0$ for every $\left(\bar{y}, Y^{\prime}\right)$ and $\left(y, y^{\prime}\right)$ in the set $R$ and every $z$. This difference is due to the difference in the method of proving the lower semi-continuity of a parametric integral from the method used in the nonparametric case.

UNIVERSity of Chicago, Chicago, Ill. 